\title{
SDU\%
}

\section{Axiomatizations of Dutta-Ray's egalitarian solution on the domain of convex games}

\author{
by \\ Pedro Calleja, Francesc Llerena and Peter Sudhölter
}

Discussion Papers on Business and Economics

No. $4 / 2020$

FURTHER INFORMATION Department of Business and Economics Faculty of Business and Social Sciences University of Southern Denmark Campusvej 55, DK-5230 Odense M 


\title{
Axiomatizations of Dutta-Ray's egalitarian solution on the domain of convex games
}

\author{
Pedro Calleja ${ }^{\mathrm{a}}$, Francesc Llerena ${ }^{\mathrm{b}}$, Peter Sudhölter ${ }^{\mathrm{c}, *}$ \\ ${ }^{a}$ Departament de Matemàtica Econòmica, Financera i Actuarial, Universitat de Barcelona-BEAT, \\ Av. Diagonal, 690, 08034 Barcelona, Spain \\ ${ }^{b}$ Departament de Gestió d'Empreses, Universitat Rovira i Virgili-CREIP, \\ Av. de la Universitat, 1, 43204 Reus, Spain \\ ${ }^{c}$ Department of Business and Economics, University of Southern Denmark, \\ Campusvej 55, 5230 Odense M, Denmark
}

\begin{abstract}
We show that on the domain of convex games, Dutta-Ray's egalitarian solution is characterized by core selection, aggregate monotonicity, and bounded richness, a new property requiring that the poorest players cannot be made richer within the core. Replacing "poorest" by "poorer" allows to eliminate aggregate monotonicity. Moreover, strengthening core selection into bilateral consistency à la Davis and Maschler, and Pareto optimality into individual rationality and bilateral consistency à la Hart and Mas-Colell, we obtain alternative and stylized axiomatic approaches.
\end{abstract}

Keywords: Dutta-Ray's egalitarian solution, axiomatizations, convex TU game

JEL: $\mathrm{C} 71$

\section{Introduction}

In the context of transferable utility cooperative games (games, for short), Dutta and Ray (1989) introduced the egalitarian solution which combines individual interests with the Lorenz criterion to promote equality. Although this solution lacks general existence properties, on the domain of convex games it selects the unique Lorenz maximal imputation within the core. On this domain, the first axiomatizations of the egalitarian solution were provided by Dutta (1990) by means of DM-consistency or HM-consistency, that is, consistency with respect to (w.r.t) the reduced games proposed by Davis and Maschler (1965) or Hart and Mas-Colell (1989), respectively, together with constrained egalitarianism $(\mathrm{CE})$, a prescriptive property that determines the solution for two player games.

\footnotetext{
*Corresponding author: calleja@ub.edu (P. Calleja), francesc.llerena@urv.cat (F. Llerena), psu@sam.sdu.dk (P. Sudhölter).
} 
Klijn et al. (2000) reformulated the above characterizations replacing CE by efficiency (EF), also known as Pareto optimality, that requires the solution to distribute the entire worth of the grand coalition, equal division stability (EDS), which forces the solution to select an allocation in the equal division core (Selten, 1972), bounded maximum payoff (BMP) imposing an upper bound for the payoffs of the players receiving most, and only requiring DM-consistency and HM-consistency when these richest players leave with their assigned payoffs. Hougaard et al. (2001) described another axiomatization combining DM-consistency and EF with non-emptiness and rich are strong (RS), requiring that the solution can only make a player $i$ richer than another player $j$ if the maximum surplus (in the sense of Davis and Maschler, 1965) of $i$ over $j$ is positive and larger than the maximum surplus of $j$ over $i$. Arin et al. (2003) reinterpreted the egalitarian solution providing a characterization without making use of any consistency property and invoking continuity, equal treatment of equals, and independence of irrelevant core allocations. ${ }^{1}$ Recently, Llerena and Mauri (2017) provided a characterization imposing DM-consistency for reduced games with at most two players and suitable well-known properties such as individual rationality (IR) or core selection (CS), which guarantee that each single player or coalition receive, respectively, at least what they can get by themselves.

In this paper, we provide two new characterizations without consistency. To do so, we use aggregate monotonicity (AM) defined by Megiddo (1974), a very natural property requiring that no player suffers if only the worth of the grand coalition increases, ${ }^{2}$ and bounded richness (BR), imposing an upper bound for the payoffs of non-poorest players, together with CS. Up to our knowledge, AM has not been employed before in any of the existing characterizations of the egalitarian solution. Strengthening BR, replacing "poorest" by "poorer", allows to eliminate AM. Moreover, we prove that on the domain of convex games DM-consistency for two-person reduced games, called bilateral DMconsistency (2-DMC), implies CS. Furthermore, we show that bilateral HM-consistency (2-HMC) together with IR imply EF. These logical implications lead to new and stylized axiomatizations. Indeed, 2-DMC together with either BMP or RS characterize the egalitarian solution. Additional axiomatizations emerge when replacing 2-DMC by 2-HMC and IR. We also show that the egalitarian solution can be characterized by constrained welfare egalitarianism (CWE) in the sense of Calleja et al. (2020) and either 2-DMC or 2-HMC and IR. We recall that CWE requires to distribute an additional amount obtained

\footnotetext{
${ }^{1}$ See Arin et al. (2003) for the precise definitions of these properties.

${ }^{2}$ See also Hougaard et al. (2005) for a generalization of Dutta-Ray's egalitarian solution on the domain of convex games satisfying monotonicity properties.
} 
by the grand coalition to the poorer players making payoffs as equal as possible subject to nobody is worse off. Hence, CWE implies AM. However, egalitarianism may regard CWE, though stronger than AM, as even more appealing. Indeed, CWE prioritizes those players who received less before the grand coalition became richer.

The remainder of the paper is organized as follows. Section 2 contains preliminaries on games. In Section 3 we introduce properties of solutions. Section 4 contains our main results. Subsection 4.1 is devoted to the characterization results of the egalitarian solution with AM and without consistency. In Subsection 4.2 we provide alternative axiomatizations for a variable society of agents making use of 2-DMC and 2-HMC.

\section{Preliminaries}

Let $U$ be a set (the universe of potential players) and $\mathcal{N}$ be the set of coalitions in $U$ (a coalition is a nonempty finite subset of $U$ ). Given $S, T \in \mathcal{N}$, we use $S \subset T$ to indicate strict inclusion, that is, $S \subseteq T$ and $S \neq T$. By $|S|$ we denote the cardinality of the coalition $S \in \mathcal{N}$. We assume that $|U| \geq 3$. Given $N \in \mathcal{N}$, let $\mathbb{R}^{N}$ stand for the set of all real functions on $N$. An element $x \in \mathbb{R}^{N}, x=\left(x_{i}\right)_{i \in N}$, is a payoff vector for $N$. For all $S \subseteq N, x(S)=\sum_{i \in S} x_{i}$, with the convention $x(\emptyset)=0$. For each $x \in \mathbb{R}^{N}$ and $T \subseteq N, x_{T}$ denotes the restriction of $x$ to $T: x_{T}=\left(x_{i}\right)_{i \in T} \in \mathbb{R}^{T}$. Given $N \in \mathcal{N}$, for all $x, y \in \mathbb{R}^{N}, x \geq y$ if $x_{i} \geq y_{i}$ for all $i \in N$. For all $\alpha \in \mathbb{R}, \alpha_{+}=\max \{0, \alpha\}$. For any two vectors $y, x \in \mathbb{R}^{N}$ with $y(N)=x(N)$, we say that $y$ weakly Lorenz dominates $x$, denoted by $y \succeq_{\mathcal{L}} x$, if $\min \{y(S)|S \subseteq N| S \mid,=k\} \geq \min \{x(S)|S \subseteq N| S \mid,=k\}$, for all $k=1,2, \ldots, n-1$. We say that $y$ Lorenz dominates $x$, denoted by $y \succ_{\mathcal{L}} x$, if at least one of the above inequalities is strict.

A transferable utility cooperative game (a game) is a pair $(N, v)$ where $N \in \mathcal{N}$ is the set of players and $v: 2^{N} \longrightarrow \mathbb{R}$ is the characteristic function that assigns to each $S \subseteq N$ a real number $v(S)$, with $v(\emptyset)=0$. Given a game $(N, v)$ and $\emptyset \neq N^{\prime} \subset N$, the subgame associated to $N^{\prime}$ is denoted by $\left(N^{\prime}, v\right)$. A game $(N, v)$ is convex if, for every $S, T \subseteq N$, $v(S)+v(T) \leq v(S \cup T)+v(S \cap T)$. We denote by $\Gamma_{U}$ the set of all convex games and, for any coalition $N \in \mathcal{N}$, by $\Gamma_{N}$ the subset of games in $\Gamma_{U}$ with $N$ as player set. By $\Gamma_{U}^{\geq 2}$ we denote the set of games in $\Gamma_{U}$ with at least two players, and by $\Gamma \subseteq \Gamma_{U}$ a generic subset of $\Gamma_{U}$. For $t \in \mathbb{R}$ and any game $(N, v)$, we denote by $\left(N, v^{t}\right)$ the game defined as $v^{t}(S)=v(S)$ for all $S \subset N$ and $v^{t}(N)=v(N)+t$. Note that $\left(N, v^{t}\right)$ remains convex if $(N, v)$ is convex and $t>0$. Any $x \in \mathbb{R}^{N}$ defines the inessential game $(N, x) \in \Gamma_{U}$ by 
$x(S)=\sum_{i \in S} x_{i}$. For $(N, v) \in \Gamma_{U}$, define

$$
\begin{aligned}
& X^{*}(N, v)=\left\{x \in \mathbb{R}^{N} \mid x(N) \leq v(N)\right\}-\text { the set of feasible payoff vectors, } \\
& C(N, v)=\left\{x \in X^{*}(N, v) \mid x(S) \geq v(S) \forall S \subseteq N\right\}-\text { the core. }
\end{aligned}
$$

A solution on $\Gamma_{U}$ is a mapping $\sigma$ that assigns an element $\sigma(N, v)$ of $X^{*}(N, v)$ to any $(N, v) \in \Gamma_{U}$. The restriction of a solution $\sigma$ to a set $\Gamma \subseteq \Gamma_{U}$ is again denoted by $\sigma$. Moreover, a solution on $\Gamma \subseteq \Gamma_{U}$ is the restriction to $\Gamma$ of some solution. Notice that, we do not consider multi-valued solution.

The following well-known lemma (the proof of which is included below for the benefit of the reader) allows to recall the formula that determines the egalitarian solution of Dutta and Ray (1989).

Lemma 1. Let $(N, v) \in \Gamma_{U}$ and denote $\mu=\max _{\emptyset \neq S \subseteq N} \frac{v(S)}{|S|}$. If $\emptyset \neq S, T \subseteq N$ are such that $v(S)=\mu|S|$ and $v(T)=\mu|T|$, then $v(S \cup T)=\mu|S \cup T|$.

Proof. Note that, by convexity of $(N, v), v(S \cup T)+v(S \cap T) \geq v(S)+v(T)=\mu(|S|+|T|)$ and, by definition of $\mu, v(S \cap T) \leq \mu|S \cap T|$. Therefore, $v(S \cup T)+\mu|S \cap T| \geq \mu(|S|+|T|)=$ $\mu(|S \cup T|+|S \cap T|)$ and, hence, $v(S \cup T) \geq \mu|S \cup T|$ so that $v(S \cup T)=\mu|S \cup T|$ by the definition of $\mu$.

Let $(N, v) \in \Gamma_{U}$ and denote

$$
\mu(v)=\max _{\emptyset \neq S \subseteq N} \frac{v(S)}{|S|} \text { and } S(v)=\bigcup\left\{S \in 2^{N} \backslash\{\emptyset\}|v(S)=\mu(v)| S \mid\right\} .
$$

By Lemma 1, $\mu(v)|S(v)|=v(S(v))$. Now, we are able to introduce the egalitarian solution of $(N, v)$, denoted by $L(N, v)$. Namely, let $\left(S_{1}, \ldots, S_{m}\right)$ be the ordered partition of $N$ that is recursively determined by the requirement that $S_{k}=S\left(v_{k}\right)$, where $S_{0}=\emptyset$ and for all $k=1, \ldots, m, N_{k}=N \backslash \bigcup_{j=0}^{k-1} S_{j}$ and $\left(N_{k}, v_{k}\right)$ is defined by $v_{k}(T)=v(T \cup$ $\left.\left(N \backslash N_{k}\right)\right)-v\left(N \backslash N_{k}\right)$ for all $T \subseteq N_{k}$. Note that $N_{1}=N, v_{1}=v$, and $\left(N_{k}, v_{k}\right) \in \Gamma_{U}$ so that $S_{k}$ is well defined. The egalitarian solution $L(N, v)=\left\{x^{*}\right\}$ is given by

$$
x_{i}^{*}=\mu\left(v_{k}\right)=\frac{v_{k}\left(S_{k}\right)}{\left|S_{k}\right|} \text { for all } i \in N_{k} \text { and all } k=1, \ldots, m .
$$

Remark 1. Let $(N, v) \in \Gamma_{U}$ and $x^{*}=L(N, v)$. The allocation $x^{*}$ satisfies the following properties:

$$
\begin{aligned}
\sum_{t=1}^{k} x^{*}\left(S_{t}\right) & =v\left(\bigcup_{t=1}^{k} S_{t}\right) \text { for all } k=1, \ldots m ; \\
x_{i}^{*} & =x_{j}^{*} \text { for all } i, j \in S_{k} \text { and all } k=1, \ldots, m ; \\
x_{i}^{*} & >x_{j}^{*} \text { for all } i \in S_{t}, j \in S_{k} \text { and all } 1 \leq t<k \leq m .
\end{aligned}
$$


Moreover, according to Theorem 3 of Dutta and Ray (1989), the egalitarian solution $L$ selects the unique core element that Lorenz dominates every other core element. That is, $x^{*} \in C(N, v)$ and $x^{*} \succ_{\mathcal{L}} y$ for all $y \in C(N, v) \backslash\left\{x^{*}\right\}$.

\section{Properties of solutions}

In the following, we introduce a number of properties on the domain of convex games. Let $\Gamma \subseteq \Gamma_{U}$. A solution $\sigma$ on $\Gamma$ satisfies

- Efficiency (EF) if for all $(N, v) \in \Gamma, \sum_{i \in N} \sigma_{i}(N, v)=v(N)$;

- Individual rationality (IR) if for all $(N, v) \in \Gamma$ and all $i \in N, \sigma_{i}(N, v) \geq v(\{i\})$;

- Core selection (CS) if for all $(N, v) \in \Gamma, \sigma(N, v) \in C(N, v)$;

- Constrained egalitarianism (CE) if for all $(N, v) \in \Gamma$ with $N=\{i, j\}, i \neq j$, such that $v(\{i\}) \leq v(\{j\}), \sigma_{j}(N, v)=\max \left\{\frac{v(N)}{2}, v(\{j\})\right\}$ and $\sigma_{i}(N, v)=v(N)-$ $\sigma_{j}(N, v)$;

- Equal division stability (EDS) if for all $(N, v) \in \Gamma$ and all $\emptyset \neq S \subseteq N$, there exists $i \in S$ with $\sigma_{i}(N, v) \geq \frac{v(S)}{|S|}$;

- Rich are strong (RS) if for all $(N, v) \in \Gamma, x_{i}<x_{j}$ implies $s_{i j}(x, v) \geq\left(s_{j i}(x, v)\right)_{+}$ where $x=\sigma(N, v)$ and, for all $k, \ell \in N, s_{k \ell}(x, v)=\max \{v(S)-x(S) \mid k \in S \subseteq$ $N$ and $\ell \notin S\}$ is called the maximum surplus of $k$ over $\ell$ at $x$;

- Aggregate monotonicity (AM) if for all $(N, v) \in \Gamma$ and all $t>0$ such that $\left(N, v^{t}\right) \in$ $\Gamma, \sigma\left(N, v^{t}\right) \geq \sigma(N, v)$

- Constrained welfare egalitarianism (CWE) if for all $(N, v) \in \Gamma$, all $t>0$ such that $\left(N, v^{t}\right) \in \Gamma$, and all $i \in N, \sigma_{i}\left(N, v^{t}\right)=\sigma_{i}(N, v)+\left(\lambda-\sigma_{i}(N, v)\right)_{+}$, where $\lambda$ is determined by $\sum_{k \in N}\left(\lambda-\sigma_{k}(N, v)\right)_{+}=t$.

EF requires to distribute the entire worth of the grand coalition. IR imposes that no single player can improve the payoff proposed by the solution without cooperating, while CS is a sort of secession-proofness property that extends this requirement to any coalition, i.e., no coalition worth is higher than the payoff to that coalition proposed by the solution. CE forces to select the egalitarian solution $L$ for two player games, which divides the worth of the grand coalition as equal as possible preserving IR. Under $\mathrm{EF}, \mathrm{EDS}$ is equivalent to imposing the solution to select a payoff vector from the equal 
division core (Selten, 1972), ${ }^{3}$ which implies that the proposed payoff vector cannot be blocked by any coalition using its equal division allocation. Note that since for convex games the core is non-empty and, moreover, it is a subsset of the equal division core, on $\Gamma \subseteq \Gamma_{U}$ CS implies EDS and EDS implies IR. RS applies to any pair of agents $i, j \in N$, and it requires that the solution may only assign a larger payoff to player $j$ compared to player $i$ if $j$ is stronger than $i$, i.e., if the maximum surplus of $j$ over $i, s_{j i}(x, v)$, is positive and larger than $j$ 's maximum surplus over $i$. AM requires that no player is worse off if only the worth of the grand coalition is increased. CWE implies to distribute an additional amount to the poorer players so that their final payoffs become equal but not larger than the remaining players' original payoffs.

The next three properties can be interpreted as solidarity requirements in the sense that they fix a maximum threshold for the payoff of some particular coalitions. Let $\Gamma \subseteq \Gamma_{U}$. A solution $\sigma$ on $\Gamma$ satisfies

- Bounded maximum payoff (BMP) if for all $(N, v) \in \Gamma, \sum_{i \in S^{\max }} \sigma_{i}(N, v) \leq v\left(S^{\max }\right)$ where $S^{\max }=\arg \max _{j \in N} \sigma_{j}(N, v)$;

- Bounded richness (BR) if for all $(N, v) \in \Gamma, \sum_{i \in N \backslash S^{m i n}} \sigma_{i}(N, v) \leq v\left(N \backslash S^{\text {min }}\right)$, where $S^{\text {min }}=\arg \min _{j \in N} \sigma_{j}(N, v)$;

- Strong bounded richness (SBR) if for all $(N, v) \in \Gamma, \sum_{i \in N \backslash S} \sigma_{i}(N, v) \leq v(N \backslash S)$ for all $\alpha \in \mathbb{R}$, where $S=\left\{i \in N \mid \sigma_{i}(N, v)<\alpha\right\}$.

BMP imposes that the payoff to the coalition of players with the highest payoff does not exceed the worth of this coalition, while BR imposes this upper bound for the payoff to the coalition of all non-poorest players. SBR strengthens BR (and also implies BMP) by replacing poorest players by players who are poorer than any wealth level $\alpha \in \mathbb{R}$.

All the aforementioned properties, except CWE, BR, and SBR, have been used before in the literature to characterize the egalitarian solution of Dutta-Ray (1989) and other solution concepts. However, these new properties are intuitive modifications of two wellknown properties. Indeed, CWE is stronger than AM, but may be regarded as even more appealing form an egalitarian point of view. Moreover, the remaining new properties BR and SBR arise by modifying BMP in two natural ways.

\footnotetext{
${ }^{3}$ For any game $(N, v)$, the equal division core is defined by $E D C(N, v)=\left\{x \in \mathbb{R}^{N} \mid x(N)=v(N)\right.$ and $\forall \emptyset \neq S \subseteq N$ there is $i \in S$ such that $\left.x_{i} \geq \frac{v(S)}{|S|}\right\}$.
} 
Now, we recall consistency properties that refer to suitable notions of reduced games. Let $\Gamma \subseteq \Gamma_{U}$. A solution $\sigma$ on $\Gamma$ satisfies

- DM-consistency (DMC) if for all $(N, v) \in \Gamma$ and all $\emptyset \neq S \subset N,\left(S, v_{S, x}\right) \in \Gamma$ and $\sigma\left(S, v_{S, x}\right)=x_{S}$ where $x=\sigma(N, v)$ and $\left(S, v_{S, x}\right)$ is the game defined by

$$
v(T)=\max _{Q \subseteq N \backslash S}\{v(T \cup Q)-x(Q)\} \text { for all } \emptyset \neq T \subset S
$$

and

$$
v_{S, x}(S)=v(N)-x(N \backslash S){ }^{4}
$$

- HM-consistency (HMC) if for all $(N, v) \in \Gamma$ and all $\emptyset \neq S \subset N,\left(S, v_{S, \sigma}\right) \in \Gamma$ and $\sigma\left(S, v_{S, \sigma}\right)=x_{S}$ where $x=\sigma(N, v)$ and $\left(S, v_{S, \sigma}\right)$ is the game defined by

$$
v_{S, \sigma}(T)=v(T \cup(N \backslash S))-\sum_{i \in N \backslash S} \sigma_{i}(T \cup(N \backslash S), v) \text { for all } \emptyset \neq T \subseteq S .^{5}
$$

The bilateral DM-consistency (2-DMC) and bilateral HM-consistency (2-HMC) only require DMC and HMC when $|S|=2$, respectively.

Note that if the solution is consistent and assigns $x$ to a game $(N, v)$, then, for every coalition $S \subseteq N$, the payoff allocation $x_{S}$ solves the corresponding reduced game w.r.t. $S$ and, therefore, it is consistent with the expectation of the members of $S$ as reflected by this reduced game. The above definitions are due to Sobolev (1975) and Hart and MasColell (1989), respectively. The egalitarian solution $L$ satisfies DMC on $\Gamma_{U}$. However, as was shown by Hokari (2002), it satisfies 2-HMC but violates HMC on $\Gamma_{U} \cdot{ }^{6}$

\section{Characterization results}

This section is divided into two parts. First, we characterize the egalitarian solution by means of $\mathrm{AM}$ and for a fixed society of agents. In the second part, we use consistency properties. Remarkably, we show that 2-DMC implies CS and 2-HMC together with IR imply EF. These logical consequences allow us to obtain new and compacted axiomatic approaches.

\footnotetext{
${ }^{4}$ The game $\left(S, v_{S, x}\right)$ is called DM-reduced game of $(N, v)$ w.r.t. $S$ at $x$ and was introduced by Davis and Maschler (1965).

${ }^{5}$ The game $\left(S, v_{S, \sigma}\right)$ is called the HM-reduced game of $(N, v)$ w.r.t. $S$ at $\sigma$ and was introduced by Hart and Mas-Colell (1989). Note that the set of convex games $\Gamma_{U}$ is closed under taking subgames.

${ }^{6}$ Indeed, Hokari's (2002) Example 1 shows that the HM-reduced game of a convex game may not be convex.
} 


\subsection{Characterizations of the egalitarian solution without consistency}

Our first characterization result makes use of CS, AM, and BR.

Theorem 1. Let $N \in \mathcal{N}$. The egalitarian solution $L$ on $\Gamma_{N}$ is the unique solution that satisfies $\mathrm{CS}, \mathrm{AM}$, and $\mathrm{BR}$.

Proof. It is well known that the egalitarian solution $L$ satisfies CS and AM. Note that CS implies EF. By Remark 1 it also satisfies BR. To show uniqueness, let $\sigma$ be a solution satisfying these properties. Let $(N, v) \in \Gamma_{N}$. Denote $x=\sigma(N, v)$. By CS, $x \in C(N, v)$. Let $x^{*}=L(N, v), m, S_{0}, S_{k}, N_{k}$ and $\left(N_{k}, v_{k}\right)$ for $k=1, \ldots, m$ be defined in equation (1) and the preceding paragraph. It remains to show that $x=x^{*}$. Let $\alpha=\min \left\{x_{i} \mid i \in N\right\}$ and $S=\left\{i \in N \mid x_{i}=\alpha\right\}$. We proceed by induction on $m$.

If $m=1$ then, by EF of $x$ and $x^{*}, \alpha \leq \frac{v(N)}{|N|}=x_{j}^{*}$ for all $j \in N$. Hence, by BR and $\mathrm{CS}, v(N \backslash S)=x(N \backslash S)=v(N)-x(S) \geq v(N)-x^{*}(S)=x^{*}(N \backslash S)$. We conclude that $x(S)=x^{*}(S), S=N$ and $x=x^{*}$.

Induction hypothesis: $\sigma(N, v)=L(N, v)$ whenever $m<\ell$ for some $\ell \in \mathbb{N}, \ell>1$.

We now assume that $m=\ell$. Put

$$
t=\left|S_{m}\right|\left(\frac{v_{m-1}\left(S_{m-1}\right)}{\left|S_{m-1}\right|}-\frac{v_{m}\left(S_{m}\right)}{\left|S_{m}\right|}\right)>0
$$

and observe that $y^{*} \in \mathbb{R}^{N}$ defined by $y_{i}^{*}=\max \left\{x_{i}^{*}, v_{m-1}\left(S_{m-1}\right) /\left|S_{m-1}\right|\right\}$ for all $i \in N$ is the egalitarian solution of $\left(N, v^{t}\right)$. Hence, by induction hypothesis, $\sigma\left(N, v^{t}\right)=y^{*}$. By $\mathrm{AM}, x \leq y^{*}$. By CS, Remark 1 implies $x_{i}=x_{i}^{*}$ for all $i \in N \backslash S_{m}$. By EF, $\alpha \leq x_{i}^{*}$ for all $i \in N$. Hence, by BR and CS, $v(N \backslash S)=x(N \backslash S)=v(N)-x(S) \geq v(N)-x^{*}(S)=$ $x^{*}(N \backslash S)=v(N \backslash S)$. We conclude that $\alpha=\min \left\{x_{i}^{*} \mid i \in N\right\}$ and, hence, $S=S_{m}$ and $x=x^{*}$.

Remark 2. The following examples show that each of the properties employed in Theorem 1 is logically independent of the remaining properties, provided $|N| \geq 3$. Clearly, if $|N| \leq 2$, BR and CS imply CE and, consequently, AM.

(i) The equal split solution $E D$, defined by $E D_{i}(N, v)=v(N) /|N|$ for all $i \in N$ and all games $(N, v)$, satisfies $\mathrm{AM}$ and $\mathrm{BR}$ but not $\mathrm{CS}$.

(ii) Let $i, j$ be two distinct elements of $N$ and define the game $(N, u)$ by $u(S \cup\{i, j\})=1$ and $u(S)=u(S \cup\{i\})=u(S \cup\{j\})=0$ for all $S \in N \backslash\{i, j\}$. Now define the solution $\sigma$ as follows: $\sigma(N, v)=L(N, v)$ for all $(N, v) \in \Gamma_{N}$ with $v \neq u$ and $\sigma_{i}(N, u)=2 / 3, \sigma_{j}(N, u)=1 / 3, \sigma_{k}(N, u)=0$ for all $k \in N \backslash\{i, j\}$. Then, $\sigma$ satisfies CS and BR (because $|N|>2$ ), but not AM. 
(iii) Let $\prec$ be a strict total order on $N$ and $\preceq$ its reflexive cover. For all $(N, v) \in \Gamma_{N}$ and all $i \in N$ define the marginal contribution solution relative to $\prec$ as follows: $m c_{i}^{\prec}(N, v)=v(\{j \in N \mid j \preceq i\})-v(\{j \in N \mid j \prec i\})$. Then, $m c^{\prec}$ satisfies CS and AM but not BR.

We now show that CS and SBR imply AM. Hence, these two properties alone characterize the egalitarian solution $L$.

Theorem 2. Let $N \in \mathcal{N}$. The egalitarian solution $L$ on $\Gamma_{N}$ is the unique solution that satisfies $\mathrm{CS}$ and $\mathrm{SBR}$.

Proof. Indeed, $L$ satisfies CS and SBR by Remark 1 . To show uniqueness, let $\sigma$ be a solution satisfying CS and SBR. Let $(N, v) \in \Gamma_{N}$. Denote $x=\sigma(N, v)$ and let $x^{*}=$ $L(N, v), m, S_{0}, S_{k}, N_{k},\left(N_{k}, v_{k}\right)$ for $k=1, \ldots, m$ be defined in equation (1) and the preceding paragraph. It remains to show that $x=x^{*}$. Assume, on the contrary, $x \neq x^{*}$. Let $m$ be minimal such that there exists $i \in S_{m}$ with $x_{i}<x_{i}^{*}=: \alpha$. Let $S=\{j \in$ $\left.N \mid x_{j}<\alpha\right\}$ and $T=N \backslash S$. Hence $T \supseteq \bigcup_{k=1}^{m-1} S_{k}$ and $x_{j} \geq x_{j}^{*}$ for all $j \in T$. By SBR and CS, $x(T)=v(T)=x^{*}(T)$, hence $x_{j}=x_{j}^{*} \geq \alpha$ for all $j \in T$. As $i \in S_{m} \backslash T$, $T \subset \bigcup_{k=1}^{m} S_{k}$, hence $x\left(\bigcup_{k=1}^{m} S_{k}\right)<x^{*}\left(\bigcup_{k=1}^{m} S_{k}\right)=v\left(\bigcup_{k=1}^{m} S_{k}\right)$, and a contradiction to $\mathrm{CS}$ is obtained.

Remark 3. The following examples show that each of the properties employed in Theorem 2 is logically independent of the remaining properties, provided $|N| \geq 2$.

(i) The equal split solution $E D$ satisfies SBR but not CS.

(ii) The marginal contribution solution $m c^{\prec}$ satisfies CS but not SBR.

\subsection{Characterizations of the egalitarian solution with consistency}

For our axiomatizations of the egalitarian solution based on Davis-Maschler consistency we need two lemmas. The first lemma shows that 2-DMC implies CS on the domain of convex games with at least two players.

Lemma 2. If the solution $\sigma$ on $\Gamma_{\bar{U}}^{\geq 2}$ satisfies 2-DMC, then it satisfies $\mathrm{CS}$ as well.

Proof. Let $(N, v) \in \Gamma_{\bar{U}}^{\geq 2}$. We consider two cases:

(i) $|N|=2$. By the assumption $|U| \geq 3$ there exists $k \in U \backslash N$. Let $M=N \cup\{k\}$ and $(M, w)$ be the game that arises from $(N, v)$ by adding the null player $k$, i.e., $w$ is given by $w(S)=v(S \cap N)$ for al $S \subseteq M$. Note that $(M, w)$ is still convex. 
Claim: If $(N, v)$ is inessential, then $\sigma(N, v)$ is the unique element of $C(N, v)$. In order to show the claim, note that $(M, w)$ is inessential. Let $y \in \mathbb{R}^{M}$ be defined by $y_{i}=w(\{i\})$ for all $i \in M$, hence $y(S)=w(S)$ for all $S \subseteq M$. Moreover, let $x=\sigma(M, w)$. For any $i \in M$, by the definition of the Davis-Maschler reduced game, $w_{M \backslash\{i\}, x}(\{j\}) \geq w(\{i, j\})-x_{i}=y(\{i, j\})-x_{i}$ for both $j \in M \backslash\{i\}$ and $w_{M \backslash\{i\}, x}(M \backslash\{i\})=w(M)-x_{i}=y(M)-x_{i}$. By 2-DMC, $\left(M \backslash\{i\}, w_{M \backslash\{i\}, x}\right)$ is convex so that $\sum_{j \in M \backslash\{i\}} w_{M \backslash\{i\}, x}(\{j\}) \leq w_{M \backslash\{i\}, x}(M \backslash\{i\})$. We conclude that

$$
\sum_{j \in M \backslash\{i\}}\left[y(\{i, j\})-x_{i}\right]=y(M)+y_{i}-2 x_{i} \leq y(M)-x_{i},
$$

hence $x_{i} \geq y_{i}$ for all $i \in M$. Now, as $x(M) \leqslant w(M)=y(M)$, we have $x=y$. Finally, since $\left(N, w_{N, y}\right)=(N, v)$, by 2-DMC, $x_{N}=y_{N}=\sigma(N, v)$ with $C(N, v)=$ $\left\{x_{N}\right\}$.

Now let $x=\sigma(M, w), i \in N$, and $N=\{i, j\}$. By 2-DMC, $\left(M \backslash\{i\}, w_{M \backslash\{i\}, x}\right)$ is convex and $x_{M \backslash\{i\}}=\sigma\left(M \backslash\{i\}, w_{M \backslash\{i\}, x}\right)$. By definition of the Davis-Maschler reduced game,

$$
\begin{gathered}
\left.w_{M \backslash\{i\}, x}(\{j\})=\max \left\{w(\{j\}), w(\{i, j\})-x_{i}\right)\right\}=\max \left\{v(\{j\}), v(N)-x_{i}\right\}, \\
w_{M \backslash\{i\}, x}(\{k\})=\max \left\{w(\{k\}), w(\{i, k\})-x_{i}\right\}=\max \left\{0, v(\{i\})-x_{i}\right\},
\end{gathered}
$$

and

$$
w_{M \backslash\{i\}, x}(M \backslash\{i\})=w(M)-x_{i}=v(N)-x_{i}
$$

so that 2-DMC implies $v(N)-x_{i} \geq \max \left\{v(\{j\}), v(N)-x_{i}\right\}+\max \left\{0, v(\{i\})-x_{i}\right\}$. Hence, $x_{i} \geq v(\{i\})$ and $v(N)-x_{i} \geq v(\{j\})$. We conclude that $\left(M \backslash\{i\}, w_{N \backslash\{i\}, x}\right)$ is inessential and thus, by 2-DMC and our claim, $x_{j}=v(N)-x_{i}$ and $x_{k}=0$. Therefore, $x_{N} \in C(N, v)$ and the proof is finished by 2-DMC.

(ii) $|N| \geq 3$. Let $x=\sigma(N, v)$ and assume that $x \notin C(N, v)$. If $x(N)<v(N)$ select any $S \subseteq N$ with $|S|=2$. By 2-DMC, $\left(S, v_{S, x}\right) \in \Gamma_{\bar{U}}^{\geq 2}$ and $x_{S}=\sigma\left(S, v_{S, x}\right)$. Now $v_{S, x}(S)=v(N)-x(N \backslash S)>x(S)$ so that $x_{S} \notin C\left(S, v_{S, x}\right)$ which contradicts case (i). Therefore, we may assume that $x(N)=v(N)$ and $x(T)<v(T)$ for some $\emptyset \neq T \varsubsetneqq N$ so that there exist $i \in T$ and $j \in N \backslash T$. Let $S=\{i, j\}$ and observe that $v_{S, x}(\{i\}) \geq v(T)-x(T \backslash\{i\})>x_{i}$ by definition of the Davis-Maschler reduced game. Therefore $x_{S}$ is not individually rational for $\left(S, v_{S, x}\right)$ and the desired contradiction is obtained by 2 -DMC and case (i). 
Remark 4. Lemma 2 does not hold on the domain of all convex games, including all 1-person games. Indeed, let $(N, v) \in \Gamma_{U}$ and $\varepsilon>0$. Define the single-valued solution $\rho$ as follows: $\rho(N, v)=L(N, v)$ if $|N| \geq 2$, and $\rho(N, v)=v(N)-\varepsilon$ otherwise. Then, $\rho$ satisfies 2-DMC but not CS.

Since, on $\Gamma_{U}^{\geq 2}$, CS implies EF, EDS, and IR, an immediate consequence of Lemma 2 is the following corollary.

Corollary 1. If the solution $\sigma$ on $\Gamma_{\bar{U}}^{\geq 2}$ satisfies 2 -DMC, then it satisfies EF, EDS, and IR as well.

The second lemma shows that IR and CWE imply CE.

Lemma 3. If the solution $\sigma$ on $\Gamma_{U}$ satisfies IR and CWE, then it satisfies CE as well.

Proof. Let $(N, v)$ be a two person convex game with $N=\{i, j\}, i \neq j$ and $v(\{i\}) \leq$ $v(\{j\})$. Let $t=v(N)-v(\{i\})-v(\{j\}) \geq 0$. By IR and CWE, for all $k \in N, \sigma_{k}(N, v)=$ $\max \{\lambda, v(\{k\})\}$, where $\lambda \in \mathbb{R}$ is determined by $\sum_{k \in N}(\lambda-v(\{k\}))_{+}=t$. If $t>v(\{j\})-$ $v(\{i\})$, then $\lambda=\frac{v(N)}{2}>v(\{j\}) \geq v(\{i\})$ and hence $\sigma_{j}(N, v)=\sigma_{i}(N, v)=\frac{v(N)}{2}$. If $t \leq v(\{j\})-v(\{i\})$, then $\lambda=v(N)-v(\{j\})<v(\{j\})$ and thus $\sigma_{j}(N, v)=v(\{j\})$ and $\sigma_{i}(N, v)=v(N)-v(\{j\})$. In both cases, $\sigma(N, v)=C E(N, v)$.

Lemma 3.2 of Klijn et al. (2000) shows that EF, EDS, and BMP imply CE. It is straightforward and left to the reader to check that EF, IR, and RS together also imply CE. Theorem 5.3 of Dutta (1990) characterizes the egalitarian solution $L$ by means of $\mathrm{CE}$ and DMC. In fact, in the uniqueness part of his proof, Dutta only used 2-DMC rather than DMC. Moreover, Calleja et al. (2020) showed that the egalitarian solution $L$ satisfies CWE. Combining these results with Corollary 1 and Lemma 3 we obtain the following new characterizations.

Corollary 2. On the domain $\Gamma_{\bar{U}}^{\geq 2}$,

(i) the egalitarian solution $L$ is the unique solution that satisfies 2-DMC and BMP.

(ii) the egalitarian solution $L$ is the unique solution that satisfies 2-DMC and $\mathrm{RS}$.

(iii) the egalitarian solution $L$ is the unique solution that satisfies 2-DMC and CWE.

Remark 5. Each of the properties in Corollary 2 is logically independent of the remaining properties, even for two-person games (i.e., for $|U|=2$ ).

(i) The equal split solution ED satisfies BMP, RS, and CWE but not 2-DMC. 
(ii) Let $(N, v) \in \Gamma_{\bar{U}}^{\geq 2}$. Schmeidler's (1969) (pre-)nucleolus, $\nu(N, v),{ }^{7}$ satisfies 2-DMC but neither BMP nor RS nor CWE.

Note that, in view of Remark 4, none of the characterizations of $L$ presented in Corollary 2 hold when expanding the domain of convex games with at least 2 players to the domain of all convex games, including all 1-person games. However, imposing 2-HMC instead of 2-DMC and employing IR as an additional property would yield axiomatizations on the domain of all convex games.

The rest of this section is devoted to the question to what extent the characterizations in Corollary 2 still hold if we replace 2-DMC by 2-HMC. We do not know if, on the domain of convex games with at least two players, 2-HMC implies CS. However, on the full domain of convex games, if we additionally impose IR, then we can show that EF is also satisfied. We finally deduce that we can replace 2-DMC by 2 -HMC in the modified version of Corollary 2 that works on the domain of all convex games when employing IR in addition.

Lemma 4. If the solution $\sigma$ on $\Gamma_{U}$ satisfies IR and 2-HMC, then it satisfies $\mathrm{EF}$ as well.

Proof. Let $(N, v) \in \Gamma_{U}$. If $|N|=1$, the proof is finished by IR (and feasibility).

If $|N|=2$, by the assumption $|U| \geq 3$ there exists $k \in U \backslash N$. Let $M=N \cup\{k\}$ and $(M, w)$ be the game that arises from $(N, v)$ by adding the null player $k$, i.e., $w$ is given by $w(S)=v(S \cap N)$ for al $S \subseteq M$. Note that $(M, w)$ is still convex. Recall that, if $(N, v)$ is inessential, then $\sigma(N, v)$ is the unique element of $C(N, v)$ by IR (and feasibility). Let $x=\sigma(M, w), i \in N$, and $N=\{i, j\}$. Then $w_{M \backslash\{i\}, \sigma}(\{j\})=v(N)-\sigma_{i}(N, v)$ and $w_{M \backslash\{i\}, \sigma}(\{k\})=v(\{i\})-\sigma_{i}(\{i, k\}, w)=0$, where the last equation follows because $(\{i, k\}, w)$ is inessential. By IR and 2-HMC, $x_{j} \geq v(N)-\sigma_{i}(N, v)$ and $x_{k} \geq 0$. Let $y=\sigma(N, v)$. As $y(N) \leq v(N), x_{j} \geq v(N)-y_{i}$ and, analogously, $x_{i} \geq v(N)-y_{j}$, we have $v(N) \geq x(M) \geq 2 v(N)-y(N)+x_{k} \geq v(N)+x_{k} \geq v(N)$ so that all inequalities must be equations, i.e., $x_{i}+x_{j}=v(N)$ and $x_{k}=0$. Since $(\{i, k\}, w)$ and $(\{j, k\}, w)$ are inessential, by IR (and feasibility) $\sigma_{k}(\{i, k\}, w)=\sigma_{k}(\{j, k\}, w)=0$ and thus $\left(N, w_{N, \sigma}\right)=(N, v)$. Finally, by 2-HMC we conclude that $x_{N}=\sigma\left(N, w_{N, \sigma}\right)=\sigma(N, v)$ is efficient.

If $|N| \geq 3$, assume that $x=\sigma(N, v)$ satisfies $x(N)<v(N)$. Then, for any $S \subseteq N$ with $|S|=2, x(S)<v_{S, \sigma}(S)=v(N)-x(N \backslash S)$, a contradiction.

As we have seen before, EF, IR, and either BMP or RS or CWE imply CE. Theorem 5.4 of Dutta (1990) stating that CE and HMC characterize the egalitarian solution $L$ is

\footnotetext{
${ }^{7}$ That is, the unique feasible payoff vector that lexicographically minimizes the non-increasingly ordered vector of excesses $(v(S)-x(S))_{S \subseteq N}$ over the set of feasible payoff vectors.
} 
not entirely correct as shown by Hokari (2002). In fact, $L$ does not satisfy HMC because a HM-reduced game of a convex game is not necessarily convex. However, the following mild modification of the foregoing theorem holds. A careful inspection of Dutta's proof shows that HM-reduced games of convex games have a non-empty core. Hence, twoperson HM-reduced games are convex. Therefore, $L$ satisfies 2-HMC on the domain of convex games. Also, as in the case of Dutta's characterization of $L$ with DMC, the uniqueness proof only uses 2-HMC rather than HMC. Hence, $L$ is characterized by $\mathrm{CE}$ and 2-HMC.

These observations, together with Lemma 4, lead to the following characterizations.

Corollary 3. On the domain $\Gamma_{U}$,

(i) the egalitarian solution $L$ is the unique solution that satisfies 2-HMC, IR, and BMP.

(ii) the egalitarian solution $L$ is the unique solution that satisfies $2-\mathrm{HMC}$, IR, and RS.

(iii) the egalitarian solution $L$ is the unique solution that satisfies 2-HMC, IR, and CWE.

Remark 6. Each of the properties in Corollary 3 is logically independent of the remaining properties.

(i) The single-valued solution $\rho$ as defined in Remark 4 satisfies 2-HMC, BMP, RS, and CWE but not IR.

(ii) Let $(N, v) \in \Gamma_{U}$. The convex root game of $(N, v)$, denoted by $\left(N, v_{r}\right)$, is the convex game with the smallest worth of the grand coalition such that $v_{r}(S)=v(S)$ for all $S \subset N$. That is, $\left(N, v_{r}\right)=\left(N, v^{\tau}\right)$ where $\tau \in \mathbb{R}$ is such that $\left(N, v^{t}\right) \notin \Gamma_{U}$ for all $t<\tau$. Note that $\left(N, v_{r}\right)$ is well defined since $v_{r}(N)=\max \{v(S)+v(T)-v(S \cap$ $T) \mid S, T \subseteq N, S \cup T=N\}$.

- For all $(N, v) \in \Gamma_{U}$ define the solution $\sigma$ as follows: $\sigma(N, v)=L\left(N, v_{r}\right)$. Then, $\sigma$ satisfies IR, BMP, and RS, but not 2-HMC.

- For all $(N, v) \in \Gamma_{U}$ define the solution $\beta$ as follows: $\beta(N, v)=\nu(N, v)$ if $(N, v)=\left(N, v_{r}\right)$, and $\sigma_{i}(N, v)=\nu_{i}\left(N, v_{r}\right)+\left(\lambda-\nu_{i}\left(N, v_{r}\right)\right)_{+}$otherwise, where $\lambda \in \mathbb{R}$ is determined by $\sum_{i \in N}\left(\lambda-\nu_{i}\left(N, v_{r}\right)\right)_{+}=v(N)-v_{r}(N)$. Then, $\beta$ satisfies CWE but not 2-HMC.

(iii) Let $\prec$ be a strict total order on $U$. The marginal contribution solution $m c^{\prec}$ satisfies 2-HMC and IR, but neither BMP nor RS nor CWE. 


\section{Acknowledgements}

The first two authors acknowledge support from research grants ECO2016-75410P(AEI/FEDER,UE) and ECO2017-86481-P(AEI/FEDER,UE), the second author also acknowledges support from Universitat Rovira i Virgili and Generalitat de Catalunya under projects 2017PRF-URV-B2-B3 and 2017SGR770, and the third author acknowledges support from research grant ECO2015-66803-P(MINECO/FEDER).

\section{References}

\section{References}

[1] Arin, J., J. Kuipers, and D. Vermeulen (2003) Some characterizations of egalitarian solutions on classes of TU-games. Mathematical Social Sciences, 46: 327-345.

[2] Calleja, P., F. Llerena, and P. Sudhölter (2020) Constrained welfare egalitarianism in surplussharing problems. Discussion Paper on Business and Economics N ${ }^{\circ} 1 / 2020$, University of Southern Denmark.

[3] Davis, M., and M. Maschler (1965) The kernel of a cooperative game. Naval Research Logistics Quarterly, 12: 223-259

[4] Dutta, B. (1990) The egalitarian solution and reduced game properties in convex games. International Journal of Game Theory, 19: 153-169.

[5] Dutta, B., and D. Ray (1989) A concept of egalitarianism under participation constraints. Econometrica, 57: 615-635.

[6] Hart, S., and A. Mas-Colell (1989) Potential, Value, and Consistency. Econometrica, 57: 589-614.

[7] Hokari, T. (2002) Monotone-path Dutta-Ray solutions on convex games. Social Choice and Welfare, 19: $825-844$.

[8] Hougaard, J.L., B. Peleg, and L. Thorlund- Petersen (2001) On the set of Lorenz-maximal imputations in the core of a balanced game. International Journal of Game Theory, 30: 147-165.

[9] Hougaard, J.L., B. Peleg, and L. P. Osterdal (2005) The Dutta-Ray solution on the class of convex games: A generatization and monotonicity properties. International Game Theory Review, 7: 431442 .

[10] Klijn, F., M. Slikker, S. Tijs, and J. Zarzuelo (2000) The egalitarian solution for convex games: some characterizations. Mathematical Social Sciences, 40: 111-121.

[11] Llerena, F., and L. Mauri (2017) On the existence of the Dutta-Ray's egalitarian solution. Mathematical Social Sciences, 89: 92-99.

[12] Megiddo, N. (1974) On the monotonicity of the bargaining set, the kernel, and the nucleolus of a game. SIAM Journal on Applied Mathematics, 27: 355-358.

[13] Schmeidler, D. (1969) The nucleolus of a characteristic function game. SIAM Journal Appl Math, 17: $1163-1170$.

[14] Selten, R. (1972) Equal share analysis of characteristic function experiments. In H. Sauermann, editor, Beiträge zur Experimentellen Wirtschaftsforschung, volume 6, pages 130-165, J. C. B. Mohr, Tübingen.

[15] Sobolev, A. I. (1975) The characterization of optimality principles in cooperative games by functional equations. In N. N. Vorobiev, editor, Mathematical Methods in the Social Sciences, volume 6, pages 95-151, Vilnius. Academy of Sciences of the Lithuanian SSR. in Russian. 